\title{
Survey on Penalty Charging Systems for Indian Number-plates
}

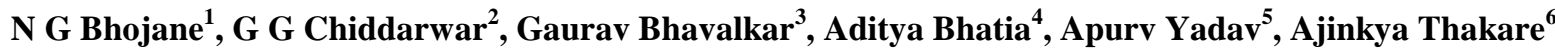 \\ Assistant Professor, Department of Computer Engineering, Sinhgad College of Engineering, Pune, India, ${ }^{1,2}$ \\ Student, Department of Computer Engineering, Sinhgad College of Engineering, Pune, India ${ }^{3,4,5,6}$
}

\begin{abstract}
In our daily life, we come across many problems caused due to traffic rule violation by some people. Mgovernance is a sub-domain of e-governance. Electronic services are made available to people because of mgovernance, with help of mobile phones. Mobile services are cheap and easily accessible in rural areas of India as well. This project strives towards improving the traffic discipline by proposing advanced technological solutions. In this project, we are designing a system which will help the traffic police officer to penalize the vehicle driver for violation of traffic rules. The penalty will be imposed to the vehicle owner if the PUC of the vehicle has expired, if the car is standing on a zebra crossing, for illegal signal crossing, crossing the prescribed speed limit and parking the car in no parking zone. The traffic police officer will have to click a picture of the vehicle number plate and select the type of complaint. A complaint will be registered against the vehicle owner and he/she will be informed about the complaint details via SMS service. The vehicle owner will be required to pay his/her fines within a stipulated time period.
\end{abstract}

Keywords: Android Based, m-governance, ANPR, Penalty System, Traffic Police, Law Enforcement.

\section{INTRODUCTION}

In the past few years, traffic congestion has increased enormously. Though the vehicle volume has increased exponentially, the road infrastructure is not yet up to the mark. This in turn leads to increased traffic congestion and road accidents. The Delhi Traffic Police's data for the year 2014 (till March '15) given below, reveals that most of the guilty drivers are violating traffic rules as they aren't much bothered about the paltry fines and negligible punishment.

\begin{tabular}{|c|c|c|}
\hline OFFENCE & 2013 & 2014 \\
\hline DRIVING WITHOUT LICENCE & 1,900 & 2,800 \\
\hline TRIPLE-RIDING & 12,800 & 13,900 \\
\hline WITHOUT HELMET & 78,100 & $1,06,800$ \\
\hline IMPROPER PARKING & 96,400 & $1,74,800$ \\
\hline DANGEROUS DRIVING & 37,400 & 46,500 \\
\hline STOP-LINE CROSSING & 1,800 & $\mathbf{3 7 , 0 0 0}$ \\
\hline NOT USING SEAT BELT & 43,900 & 64,000 \\
\hline USING MOBILE WHILE DRIVING & 350 & 1,170 \\
\hline WITHOUT SPEED GOVERNOR & 5 & 53 \\
\hline NOT DRIVING IN PROPER LANE & 532 & 905 \\
\hline SMOKING WHILE DRIVING & o & 92 \\
\hline
\end{tabular}

In an attempt to reduce the problems related to traffic and improve the traffic discipline, advanced technological solutions have been proposed in this paper.

The first stage would be the registration of vehicles in a database stored and maintained at the RTO server. The details include vehicle information such as vehicle number, vehicle model and colour as well as the owner information such as owner name, email id, mobile number, Driving license number etc. This database will also maintain the complaints registered against various vehicle owners and the fines to be paid by them and if paid then make the necessary change. The second stage is the traffic police registration and login. Each traffic police officer will require to register using credentials such as his/her name, email id, jurisdiction. They will then be given a username and password to login into the application to ensure authorized use/security.

Once logged in the traffic police will have the options of registering a complaint or checking the payment status and clearing/accepting a payment of a vehicle owner. To register a complaint, the traffic police officer will have to click a picture of the vehicle number plate from his camera and specify the type of complaint from the given options. The 
image will be sent to server where the vehicle number will be identified and the fine will be charged to the vehicle owner. To check the fines assigned to the vehicle, he will have to enter the vehicle number and will know the total charges corresponding to that vehicle. He can then accept payment and the database will be modified accordingly.

Once the complaint is registered the vehicle owner will be notified about the same through SMS service. He will also be informed if his/her vehicle has been towed in case of No Parking complaint and to which police station as well.

\section{RELATED WORK}

A review was done on different topics which would be a hurdle while designing the penalty system for traffic law enforcement. Some of the key ideas are described paper-wise are as follows:

\section{Anu Agarwal, SudhirGoswami [1]}

This paper proposes an efficient algorithm for recognition of Indian car license plate using morphological operation, thresholding operation, edge detection, bounding boxanalysis for number plate extraction, character segmentation and character recognition using template matching method. Input to the system isimage of the car containing plate and output is the actual characters of the plate. The image is captured in differentweather condition. As the light falling to it changes its brightness and contrast also changes which make it achallenging task. The block diagram of proposed system is depicted in the figure below. The process involves the following steps:

- Pre-processing

- License plate detection

- Character segmentation

- Character matching

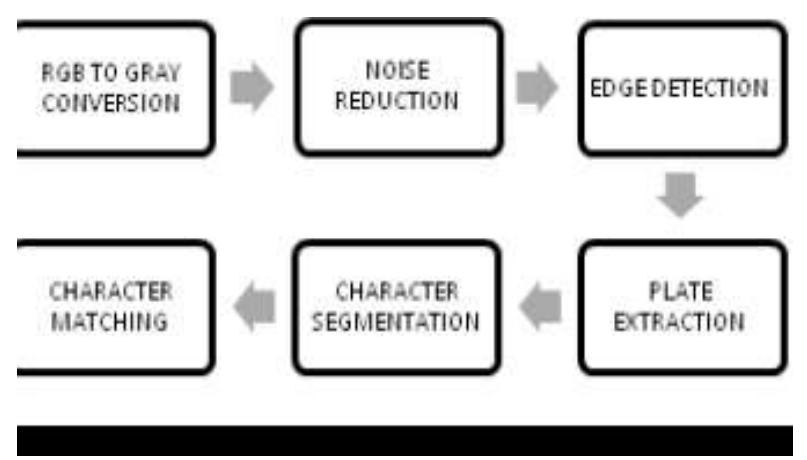

\begin{tabular}{|l|l|l|l|}
\hline S. No. & Methods Recognition & Results & Computational Time \\
\hline 1. & Morphological & Medium & Medium \\
\hline 2. & Mathematical Morphology & Low & High \\
\hline 3. & Hough+Contour & Medium & High \\
\hline 4. & Proposed Method & High & Low \\
\hline
\end{tabular}

\section{Shiv Kumar Goel, Manoj Shukla [2]}

This paper highlights various e-governance initiatives like Vahan and Sarathi for issue of driving licence and smart card. The various components of the system are:

\section{A. Vehicle Identification System:}

The Vehicle Identification System mainly captures a picture of vehicle which is violating the traffic rules on the road with the network of RFID and camera, captured images further used for various purposes as given below:

- It will be used by Registration Recognition Plate subsystem for extraction of vehicle number.

\section{B. Registration plate recognition system:}

The main purpose of Registration plate Recognition is to process the picture taken by the VIS system and extract the registration number of vehicle number plate. The registration number of the vehicle is the unique identity of the vehicle and holds the owner details.

\section{Electronic Penalty System(EPS):}

After extracting the registration number,the RTO area which the vehicle belongs to will be searched, and the owner details will be obtained. A penalty will further be imposed. After deducting the amount a SMS will be sent to the owner's registered mobile number. All transactions will be saved in a database for future reference. Data will be stored 
in the area wise database, and ultimately it will be sent to the centralized database. The architecture of EPS is divided into 4 stages: Block Level, Village Level, District Level, and State Level.

\section{Actual Working Plan:}

The VIS and registration plate systems are installed at the e-data centres which are linked with the state data centre (SDC). SDC's are connected with SWAN (State wise Area Network).All state data is connected to the Central Gateway. The cameras and RFID reader are installed on the roadside. The SDC will have the list of all registered vehicles in the state. Whenever some offence occurs, the offender's vehicle plate will be recognized, and data will be obtained via SDC. The RFID reader will extract the information of the owner with the help of data received from Vahan.

\section{Siddhartha Choubey, DrG.R.Sinha [3]}

The method practiced in this paper recognizes differences between characters by gauging the differences in pixel distribution density of different regions of alphabets and numbers.

\section{Actual methodology:}

\section{A. Pre-processing (Number Plate Extraction)}

Various pre-processing steps used are:

- $\quad$ Converting image to grey scale image,

- $\quad$ Converting image to black and white image, and

- $\quad$ Removing all objects smaller than 100 pixels.

B. Removing extra area from all regions by detection of first and last columns, done for each character and number.

Vertical scanning is done before horizontal scanning because if skew is present in the input image, then the effect of skew will be minimized. This improvement will reduce the error of first and last columns and rows. Vertical scanning (column by column) is done to detect the first and last columns for each component and cut out the area in between. This separates the license information from the background.After the first and last columns of the component have been detected, horizontal scanning is performed, to detect the first and last rows from the result of the previous step. The output of this step will contains only the characters or numbers. All extra area will be eliminated.

\section{Character and Number Recognition}

Here we use a new methodology in which the characters are separated into sub-groups on the basis of their similarity or different physical appearance.

Each group has a specific template. The template matching the input characters the most, will decide the group of the character. The subgroup is calculated on basis of indexes, by the formula:

Index of a particular template $=($ Number of pixels that matched with that template) $/$ (Totalnumber of pixels in that template).

The subgroup whose template has highest index with respect to the character is chosen as the subgroup.

Once, we get the subgroup then the physicalcharacteristics of each element that differentiate it from otherelements are used to identify the element.

\section{Case Studies on e-Governance in India 2012-2013 [4]}

Case Study on Bangalore Police:

Diligent Traffic Enforcement is a major problem across India and it is a subject of major reforms initiated by senior management of all Traffic Police establishments in India.Bangalore Traffic Police attempted to overcome the problems, by converging People, Process and Technology. Traffic enforcement is done in two stages, namely:

Spot fining- Where a violator is caught while doing violation and is slapped with a spot fine against his violation. The violator is required to pay the fine amount immediately.

Fine against notices- Where a violation is noticed and fine details are mailed to the violating vehicle's registered address, as a notice for payment.

\section{Challenges in second method:}

1. Finding addresses of vehicles registered at RTOs outside Bangalore city.

2. Finding correct $\&$ updated addresses of vehicles which frequently changed hands and ownership.

3. Getting the latest version of the registered addresses from the RTOs.

All these problems were coupled by the fact that most of the RTOs were not computerized and the mechanism of data exchange between RTO and Traffic Police was manual, sometimes leading to a backlog of 11 months. 
The third challenge was getting the citizens to pay for the violations. The problems here were primarily because of the cumbersome mechanism of payment options available with citizens.

\section{Implementation}

Under the first phase, officers' till the level of a sub-inspector were provided with handheld Blackberry devices and handheld printing devices. These devices had small application software deployed for capturing traffic violations and generating receipts against payments made. These devices were connected with a centralized server system kept at the state data centre using a GPRS / EDGE based mobile network. This helped the police officers capture\& record violations online into a central database. The second phase emphasized on process automation. Under this activity a central data repository was created at the state data centre and the central repository was connected with all police stations using a VPN network. This ensured that all violations noticed and recorded got consolidated at a central place, accessible to all police stations, which provided the citizen with an option of paying at any police station instead of the station under which the violation was recorded.

\begin{tabular}{|c|c|}
\hline Deficiencies & Cause \\
\hline Financial losses to the exchequer. & $\begin{array}{l}\text { - Lack of visibility on the field activities being performed by } \\
\text { traffic personnel. } \\
\text { - Difficulty in fixing accountability. }\end{array}$ \\
\hline $\begin{array}{l}\text { Inability to identify and track habitual } \\
\text { offenders. }\end{array}$ & $\begin{array}{l}\text { - No centralized information repository which can be used to } \\
\text { store and track violations using violating vehicle details. } \\
\text { - No institutional mechanisms either within processes or } \\
\text { through technology, which can allow capturing and } \\
\text { consolidation of violation details. }\end{array}$ \\
\hline $\begin{array}{l}\text { Inability to validate the authenticity of the } \\
\text { violation recorded. }\end{array}$ & $\begin{array}{l}\text { - Complex methodology of performing such validations, } \\
\text { which make it humanly impossible to perform such activity } \\
\text { without having some automation. } \\
\text { - Lack of full and updated vehicular data from RTOs. }\end{array}$ \\
\hline $\begin{array}{l}\text { Inability to capture latest vehicle registration } \\
\text { details from RTOs. }\end{array}$ & $\begin{array}{l}\text { - Distributed nature of database maintenance at RTOs. } \\
\text { - Lack of online connectivity with Transport Department in } \\
\text { order to receive a regular update. } \\
\text { - Manual process of data synchronization caused huge delay } \\
\text { in getting latest data. }\end{array}$ \\
\hline $\begin{array}{l}\text { Difficult and cumbersome process of citizen } \\
\text { interactions, including the process of fine } \\
\text { payments and also grievance redressal } \\
\text { mechanism. }\end{array}$ & $\begin{array}{l}\text { - Lack of centralized repository of all violations. } \\
\text { - Lack of connectivity between police stations. }\end{array}$ \\
\hline $\begin{array}{l}\text { Lack of management information system } \\
\text { required for taking key decisions. }\end{array}$ & $\begin{array}{l}\text { - No central repository of information. } \\
\text { - No definition of key performance indicators. }\end{array}$ \\
\hline
\end{tabular}

The objective was toperform the following series of activities; the activities are listed as per the priority set:

Priority I - Create a central hardware infrastructure for hosting centralized knowledge repository.

Priority II - Deploy local IT setup at each police station and connect the central hardware infrastructure with police stations.

Priority III- Create a central data repository and connect it with the local systems for regular data sharing and synchronization.

Priority IV-Develop mobile governance program by creating a mobile infrastructure by distributing handheld devices, connected to the central repository using EDGE / GPRS network, to officers on the field. This would include training to the field officers on handling and using these devices.

Priority V - Develop citizen service delivery channels, including website and payment facilitation centres.

\section{Imran Shafiq Ahmad,BoubakeurBoufama et al [5]}

This paper compares the performances of various ANPR systems by testing them on a rich vehicle database in Canada. The extracted information from various ANPR systems is essential forseveral applications, ranging from automated paymentservices like toll plazas and parking charges, to more critical applications, like border security and trafficsurveillance. The three modules considered in all ANPR systems are:

- Plate Localization,

- Character Segmentation,

- OpticalCharacter Recognition. 
The comparative study lists various methods for each of the three modules mentioned above as follows:

A. Plate localization and extraction:

The first plate extraction method is based on extracting vertical edges, by using vertical Sobel edge detector.

In the second method, after extracting gradient image, vertical histograms are usedto find the most probable strips which may contain the plate. Then, morphologicalErosion and Dilation operations are carried out to find the rectangular region of the plate.

\section{B. Character segmentation:}

The character segmentation is based on the projection ofpixels along the horizontal and vertical directions, where pixelsof the characters are projected along the vertical direction and the horizontal direction, to identifythe vertical positions and the horizontal positions of characters in the plate. This is a widely used method for character segmentation.

\section{Optical character recognition:}

The first method is basedon template matching. The given image is first binarised, with a method of adaptive thresholding. Then the pixel-wise matching is performed between the binary image of unknown characters, and the stored binarypatterns to find the most similar pattern.

The second evaluated method is based on subspaceprojection and probabilistic neural network (PNN). The PNN is trained using allcharacter instances in the character space. It has four layers:input, pattern, summation, and decision.

Input layer has the same dimension as the character space. For pattern layer, thedistance between an unknown pattern and all of the existingpatterns in each dimension are calculated using the followingequation.

$$
\text { distance }\left(x_{j}, x_{i j}\right)=\frac{1}{\sigma \sqrt{2 \pi}} \times e^{\left[-\frac{\|\| x_{j}-x_{i j} \|^{2}}{2 \sigma^{2}}\right]}
$$

Where, $x_{j}$ represents the value of the input pattern in dimension $j$, $\mathrm{x}_{\mathrm{ij}}$ represents the value of pattern iin dimension $\mathrm{j}$, and $\sigma$ isthe smoothing factor.

The summation layer adds the similarityof different dimensions for each class which is equal to theprobability of the unknown input extracted from this class.

Finally, the decision layer finds the most probable outcomeand selects the winner class.

\section{III.CONCLUSION}

M-governance, as a sub domain of E-governance proves to be really helpful to the citizens, by means of a smooth governance system with flexibility and transparency. The main idea behind developing suchtraffic enforcement systems is to quickly recognize and charge traffic offenders with a penalty and encourage them to follow traffic rules diligently and drive responsibly. Charging penalties to an offender by registering violations against the number plate of the vehicle, enables smooth enforcement and lightens the job of the traffic police. The survey done in this paper highlights the various methods used by researchers for Automatic Number Plate Recognition (ANPR) via plate localization, character segmentation and OCR. The various methods to implement fines have been discussed as well.

\section{ACKNOWLEDGEMENTS}

We are extremely thankful to our Project Guide Prof. N. G.Bhojane for his invaluable guidance and supervision that helped us in our project. He always encouraged us to explore new concepts and pursue newer dimensions to our project. We would also like to thank our Project Committee members,Prof. G. G. Chiddarwar, for giving us valuable advice on ANPR and OCR, and Prof. S. D. Wable for their suggestions, time and constant availability for valuable inputs.

\section{REFERENCES}

[1] “An Efficient Algorithm for Automatic Car Plate Detection \&Recognition”, Anu Agarwal, SudhirGoswami, IEEE2016 Second International Conference on Computational Intelligence \& Communication Technology.

[2] "Electronic Penalty an Initiative for E-Governance using RFID and Camera-based Hybrid Approach". Shiv Kumar Goel, Manoj Shukla,International Journal of Computer Applications (0975 - 8887), 2013.

[3] "Pixel Distribution Density based character recognition For Vehicle License Plate", SiddarthaChoubey, Dr. G. R. Sinha,2011 IEEE.

[4] "Case Study on Bangalore Police", Case Studies on e-Governance in India 2012-2013.

[5] "Automatic License Plate Recognition: A Comparative Study", Imran Shafiq Ahmad, BoubakeurBoufama et al, IEEE 2015 International Symposium on Signal Processing and Information Technology (ISSPIT). 